This is an electronic reprint of the original article. This reprint may differ from the original in pagination and typographic detail.

Author(s): Pasanen, Tero

Title: Violent Encounters : The Hobbesian State of Nature in DayZ

Year: $\quad 2016$

Version:

Please cite the original version:

Pasanen, T. (2016). Violent Encounters : The Hobbesian State of Nature in DayZ. In L. Joyce, \& B. Quinn (Eds.), Mapping the Digital : Cultures and Territories of Play (pp. 31-40). Inter-Disciplinary Press. http://www.inter-disciplinary.net/critical-issues/wpcontent/uploads/2014/07/pasanenvgpaper.pdf

All material supplied via JYX is protected by copyright and other intellectual property rights, and duplication or sale of all or part of any of the repository collections is not permitted, except that material may be duplicated by you for your research use or educational purposes in electronic or print form. You must obtain permission for any other use. Electronic or print copies may not be offered, whether for sale or otherwise to anyone who is not an authorised user. 


\title{
Violent Encounters: The Hobbesian State of Nature in DayZ
}

\author{
Tero Pasanen
}

\begin{abstract}
In his seminal work on social contract theory, Leviathan, Thomas Hobbes described the anarchic pre-societal condition of the state of nature. In this predicament - aptly named as "the war of all against all" - people lived poor, short and brutish lives. The dystopian world simulated in the survival horror game DayZ mirrors this natural state of man. The game invites players to roleplay survivors of a zombie apocalypse. The present paper examines the factors contributing to pervasive violence, moral ambiguity and degeneration of human condition, which are prominent features of DayZ's gameplay. The article will situate DayZ into the context of Hobbesian state of nature and explicate elements that render this hypothetical condition perpetual.
\end{abstract}

Key Words: social contract, moral ambiguity, zombie apocalypse, first-person shooters, permadeath.

$* * * * *$

\section{Welcome to Chernarus}

You spawn at the eastern sea shore of Chernarus, near the woods and quite uncertain of your location. You seek cover in the forest and wander aimlessly for a while, before arriving on a clearing. You spot a small rural village in the distance. As you move closer, you notice a group of zombies roaming around the buildings. A zombie spots you as you try to sneak pass them, giving a high pitched shriek. You rush toward the nearest accessible buildings with the aggroing zombie on your heels. The racket attracts more zombies that join the chase. You reach a two-storey house in the village centre. As you stumble through the doorway one of the pursuing zombies manages to hit you and you start to bleed. The zombie follows you slowly as you move upstairs. In one of the rooms you find a pistol. The zombie enters the room as you equip it. You fire few rounds from the hip, until you take an aimed shot to its head. The zombie falls dead. Its companions have lost their sight of you, but you have also spent almost all the ammo. Shaking from the pain you quickly bandage the wound to stop the bleeding. Your vision is tinted grey. You scour the house for more loot. Suddenly you feel the presence of another player behind you. He has heard the gunshots. He shouts "I'm friendly, I'm friendly!" on the direct voice chat, only to shoot you in point blank range. The world goes black. The screen tells the obvious: "you are dead."

The aforementioned scenario is quite common for those taking their first steps with the open world survival horror game DayZ (2012/2013). Albeit the attempt 
was short, it taught a harsh, yet a fundamental lesson about trust in this online environment.

The bleak, dystopian world simulated in DayZ mirrors the state of nature, described by Thomas Hobbes in Leviathan (1651). ${ }^{1}$ The DayZ project initially started off as an Arma 2 (2009) modification, ${ }^{2}$ before being developed into a standalone title. ${ }^{3}$ The game invites players to roleplay survivors of a zombie apocalypse. It is a nihilistic sandbox, set into a fictional post-Soviet state of Chernarus, ${ }^{4}$ where the lines between gameplay and griefing are constantly blurred and renegotiated.

This article offers a Hobbesian reading of DayZ by explicating gameplay conventions and design elements that render this hypothetical condition perpetual. ${ }^{5}$ The paper treats both the community-build mods and the standalone game.

\section{DayZ}

DayZ revolves only seemingly around the traditional survival horror paradigm, challenging the horizon of expectations when it comes to contemporary design conventions. It does not favour short learning curve or instant rewarding, and goes against the grain with its small margin for error and lack of balance. Unarmed fresh spawns are pitted against well-armed survivors, and only a small slip may cost hours of progress and investment that cannot be restored from a save point. The game has no specific winning condition, pre-defined subgoals or achievements. The sole objective is to survive as long as possible. The means to accomplish this are undetermined, left for players to sort out amongst themselves. The only operational rule is to quell thirst and hunger. Furthermore there are no rules for governing social interaction, which highlights the importance of establishing trust through communication.

Perhaps the most prominent feature in DayZ is the permanent death (permadeath) system: when a character dies it is lost for good. ${ }^{6}$ This approach is unique in online first- and third-person shooters with persistent servers. Singlespawn mechanic has been previously utilized in session-based shooters. The system has been praised - sometimes justly, sometimes idealistically - for underlining consequences made by players, for instance by invoking moral dilemmas. ${ }^{7}$ However it is painstakingly obvious that players indulge themselves with rampant acts of violence and morally questionable behaviour. This is seen in emergent use of different game features that are combined to act, for instance, as torture methods. ${ }^{8}$

The fragility of life is underlined by complex simulation of health, physical trauma, physiological needs and psychological effects. Death may be instant or prolonged, occurring when the health level drops to zero due to injuries, infection, hunger or thirst. The only solution to postpone the inescapable death is to scavenge for resources. The loot is versatile, ranging from provisions, utility items and medical supplies to weapons and vehicles. 


\section{The Self and the Other}

There are two types of alterity present in DayZ: zombies and other survivors. The zombies - otherwise known as walkers or zeds - are of twenty-first century variety. They are fast, untiring and ferocious. ${ }^{9}$ Zombies symbolize the end of mankind. They represent the posthuman condition, simultaneously being subjects and objects. ${ }^{10}$ Although possessing human features, zombies are totally stripped of individuality or virtues of humanity. These things are devoid of empathy and cannot be reasoned with. Zombies are driven only by the most primitive of priorities, the necessity to feed. Yet as adversaries they are relatively weak, predictable and easily evadable. ${ }^{11}$ For more experienced players the zeds hardly offer any challenge, even in larger groups ${ }^{12}$ Furthermore zombies only spawn as player comes within 200-300 meter radius of settlements or other loot sites. Thus they mainly act as indicators of the presence of other survivors.

The human is the apex predator. In contrast to zombies the threat posed by survivors is constant. Survivors do not represent any power structure or governmental entity that would have monopoly on the use of violence. They are individuals who try to survive the apocalypse to the best of their abilities. Survivors can be situated into the extremities of hero and bandit. All players spawn as normal survivors, but their actions alter the character state. The rule of thumb is that heroes help other survivors, whereas bandits prey on survivors. However this dichotomy is not fixed in stone. Depending on the situation a hero will murder a survivor just as easily as a bandit.

Some players have allowed themselves to be robbed or kidnapped to preserve their virtual lives. ${ }^{13}$ However these cases are quite artificial as players willingly adopt the role of the victim for the sake of immersion and role-play. The kidnappings continue only as long as the victims allow them to continue. ${ }^{14}$

\section{The State of Nature}

In his seminal work on social contract theory, Leviathan, Thomas Hobbes defined the state of nature as "war of all against all"15 (Lat. bellum omnium contra omnes) in which the life of man was "solitary, poor, nasty, brutish and short." 16 In this hypothetical pre-societal condition there were no rights, only natural freedoms. Hobbes's view of human nature was quite pessimistic. The fate of natural men was to destroy each other in a restless pursuit for power. In this context, force and fraud were considered as cardinal virtues. ${ }^{17}$

Hobbes's resolution to the state of nature was to form a commonwealth symbolised by the Leviathan - ruled by a sovereign authority wielding absolute power. ${ }^{18}$ Hobbes argued that humans ceded some of their natural freedoms to the head of the Leviathan out of fear.

The lawless dystopian circumstances simulated in DayZ mirrors the Hobbesian state of nature. The absence of predefined factions (i.e., teams) and codes of conduct result in moral ambiguity. Social interaction is governed only by implicit 
rules. There is no right or wrong as players constitute their own relative moral guidelines. In this case "the natural condition of mankind"19 is post-societal, following the collapse of civilisation.

\section{The Frames of Violence}

Similarly to Leviathan the source of competition and conflict in DayZ can be derived from the appetite for power. In a game without winning condition, defined subgoals or achievements the meaning of power is multifaceted; it can refer, for instance, to control of specific map locations, building strongholds, possessing of vehicles or valuable resources. However the use of violence is the most apparent form of power.

In theory the lack of comprehensive operational rules has potential for complex social interaction, but in reality the gameplay is too often reduced to simple deathmatching and murder for sport. As DayZ gained popularity a behavioural pattern - aptly termed as the kill-on-sight mentality (KoS) - emerged and established its position as preferred gameplay convention. ${ }^{20}$ The $\mathrm{KoS}$ is a selffeeding cycle. Those killed without an apparent reason tend to repay the favour to other players. Arbitrary acts of violence have become pervasive forms of social interaction as players assume that everyone they encounter is hostile by default. This has created an atmosphere of constant paranoia, in which others are killed for precaution rather than of spite. Firefights may erupt even if there are no initial hostile intentions as players open fire on random survivors to protect themselves.

At first glance the all-encompassing violence may seem wanton and gratuitous, but this behaviour should be situated to certain cultural frameworks that influence the player conduct. There are also design choices and technological aspects that afford this type of antisocial gameplay.

Social decay and degeneration of human condition, popularised by George Romero's Night of the Living Death (1968), are generic tropes of modern zombie fiction. $^{21}$ This established scheme creates preconceptions that steer DayZ's gameplay to specific directions, in which nihilistic, self-serving and confrontational behaviour is expected and accepted. The end of the civilization scenario that players are invited to role-play requires both villains and victims to work. These roles are not fixed, but reverse quickly as predators become prey.

The first-person shooter (FPS) genre has been player-versus-player (PvP) oriented (deathmatch or team deathmatch) since its conception. This genre convention is so deeply rooted that players seem to abide by it, even when it is not forced on them. Furthermore the ample amounts of available firearms - especially in the community-build mod versions - guide gameplay into PvP combat. Sniper rifles are not required for protection against zombies, but to ambush other survivors.

Perhaps the most fundamental reason for habitual player killing is the lack of a considerable common enemy that would force players to cooperate. Zombies 
cannot fulfil this role as they do not offer necessary challenge. Their presence as adversaries is relatively indifferent. The zeds mainly act as a reference that ties the game events to the background story. This has direct effects on meaningful of play i.e., on actions that yield purposeful outcomes. ${ }^{22}$ DayZ does not reward for killing zombies, on the contrary they only notify other survivors of your presence. They rarely carry valuable loot, compared to well-armed and equipped survivors. In addition the core mechanic of DayZ, which revolves around scavenging, is extremely repetitive and cannot maintain entertainment value for longer periods of time. Players find the essential accessories needed for survival quite quickly, and if they have already explored the map, their attention will be directed towards additional challenges, posed by other players.

Manhunt is the ultimate sport in DayZ. Waylaying others - as well as evading and surviving ambushes - is a testament of skill. Scheming and committing murder is undoubtedly the single most challenging and rewarding activity, requiring patience, timing and guile. It is an example of exercising power and asserting dominance over others.

\section{Conclusions}

DayZ is a gaming phenomenon with tremendous potential to revolutionise and reinvigorate the shooter genre, which currently suffers from a period of stagnation, represented by the generic modern military shooters (MMS) favouring instant rewarding and non-stop action. The game draws much of its allure from a delicate balance of challenge and frustration. The project is naturally laden with expectations that it cannot deliver, but it has sown a seed that can usher the genre into a new, more mature era. One of these promising elements is the permadeath system. Military shooters have been traditionally reprimanded for downplaying consequences of violence; death is a punishment from failure, but the penalty is relatively minor as characters respawn almost immediately, or the lost progress can be backtracked from savepoints. In DayZ the permadeath mechanic and complex simulation of health foreground the results of physical aggression. The system can be considered as a way to eradicate the Lazarus syndrome i.e., possibility to respawn endlessly, and counter its effects on gameplay. Along with the absence of rules that govern social interaction, the permadeath system has potential to introduce genuine moral dimension to digital gaming. ${ }^{23}$

Interestingly the deeply rooted social Darwinism is not scripted to the game, but seems to be inherent part of the gameplay itself. This demonstrates fundamental flaws in players' abilities to communicate and resolve conflicts without the use of violence. It also illustrates the power of genre conventions. One can argue that it is the prevalent egocentrism and antisocial behaviour that makes DayZ so appealing. The game can be read as a procedural social commentary ${ }^{24}$ on human nature, but perhaps more accurately it illustrates how people conduct themselves in online games. The commentary is not conveyed through the in-game 
narrative, but through collective player behaviour, resulting from the absence of social contract. The prominent kill-on-sight mentality can also be interpreted as a form of griefing, but for large segment of players it is a natural part of the game, apt to the zombie apocalypse theme. It is an inevitable consequence that follows the lack of operational and social rules.

In DayZ the perpetual state of nature is imperative as it maintains tension, enables meaningful play and creates unique atmosphere. It also situates responsibility of ethical agency on players. The emergence of a sovereign that would unite all the players under a common cause would be devastating to gameplay. The game thrives on incessant anarchy made possible by the absence of extensive social contract. The server encompassing cooperation would be the most rational way to fulfil the objective of survival, but at the same time harmonious coexistence would also be destructive to the overall experience. This is of course not to claim that all the players attempt to survive in solitude or that there is no cooperation of any kind. Like other games, DayZ involves clan activity, smaller inner groups of players and even casual ad hoc social formations. There is obvious safety in numbers, but pervasive player behavior makes cooperation extremely precarious. Furthermore the design choices and genre conventions do not support harmonious coexistence. The argument is that cooperation is not desired on the server level.

DayZ exemplifies the paradoxical logic of game controversies. The game has not generated polemic, although premeditated homicide is perhaps the most central game mechanic. It has not been dubbed as murder or torture simulator, regardless of its realistic simulation of ballistics and physical trauma or morally questionable player behaviour. The reason for this is the fictional setting, which offers more leverage and enables certain creative choices not permitted to games situated into real-world contexts. ${ }^{25}$

It remains to be seen how long DayZ can thrive on its novel game. The lack of goals and objectives, which sets the game apart from its peers, may ultimately become a burden that must be resolved in one form or another. In the end scavenging or manhunting may not be enough to maintain long-term interest. The chosen Minecraft-like development model may prove flexible enough to allow the developers to react on arising challenges as they surface.

DayZ is about the freedom of choice. At the moment the freedom to "do what thou wilt" manifests itself in violent encounters.

\section{Notes}

${ }^{1}$ Thomas Hobbes, Leviathan or the Matter, Forme \& Power of Common-Wealth and Civill (1651). 
${ }^{2}$ The initial impetus behind DayZ was to design a digital training application for the New Zealand Army. However as the idea did not stir interest in the military, it became an "entertainment-only" game.

${ }^{3}$ The original mod was developed by Dean "Rocket" Hall in 2012. In August 2012 Hall announced that DayZ would become a standalone game. The mod became a community-based effort with the release of update 1.7.3 in October 2012. This change resulted in a plethora of modifications based on the original mod. The standalone version was released in December 2013 by Bohemia Interactive with Hall working as the lead designer. In February 2014 he announced leaving the project in the end of the year. The current build of DayZ: Standalone is still in alpha stage and it is quite rudimentary compared to the community-build mod versions.

${ }^{4}$ Chernarus offers $225 \mathrm{~km}^{2}$ of playable area that breathes gloomy post-Soviet atmosphere with rural landscapes in autumn colours, populated with villages, towns and larger cities that celebrate Soviet architecture. Certain mods contribute further to the prevailing ambiance. For instance the DayZ: Taviana mod features huge statues of Lenin, billboards with communist imagery and a museum exhibiting Social Realism and communist heroes. The mod also has a Ferris wheel, which is a reference to the iconic symbol of desolation, found in the city of Pripyat at Chernobyl Exclusion Zone.

5 The observations made in this article are based on extensive participatory observation that allowed systematic experimentation with different gameplay tactics and strategies. Furthermore user-generated content, such as let's play videos, online blogs and forum discussions, were analysed.

${ }^{6}$ We can liken the permadeath system to the one-session game of narration (OSGON) model proposed by Gonzalo Frasca (2001). Similarly to the mechanic adopted by DayZ, death in the OSGON model was irreversible. However in an OSGON dying would be difficult as it is a single-player game that can only be played once.

7 Marcus Carter, Martin Gibbs and Greg Wadley, 'Death and Dying in DayZ,' Proceedings of the $9^{\text {th }}$ Australasian Conference on Interactive Entertainment: Matters of Life and Death, Article No. 22 (New York: ACM, 2013), 3.

${ }^{8}$ Handcuffed players are being force fed with disinfectant to cause chemical poisoning, which can lead to death if not cured. Initially handcuffs were design as a non-lethal method to pacify players, whereas force feeding can save unconscious players from starving or dying of thirst.

${ }^{9}$ Kyle Bishop, 'Raising the Dead: Unearthing the Non-Literary Origins of Zombie Cinema,' Journal of Popular Film and Television 33.4 (2006), 24.

${ }^{10}$ Sarah Juliet Lauro and Karen Lauro, 'A Zombie Manifesto: The Nonhuman Condition in the Era of Advanced Capitalism,' Boundary 235.1 (2008): 85-108. 
${ }^{11}$ Zombies can be easily evaded by moving in crouch or crawl position as these stances lower character's visibility and audibility. Even aggroing zombies can be eluded quite effortlessly by escaping through buildings as a technical limitation of the game engine forces running zombies to walk indoors. Breaking the line of sight causes zombies to abandon the chase.

12 The community-build mods, DayZ: Origins and DayZ: Mercenary, have increased the level of challenge and need for cooperation by implementing wellarmed AI survivors that patrol the map and hunt for players.

${ }^{13}$ Adam Ruch, 'Stockholm Syndrome: How Six Men Kidnapped Me in DayZ,' Games On Net, 5 July 2012, viewed 16 May 2014, http://games.on.net/2012/07/stockholm-syndrome-how-six-men-kidnapped-mein-dayz/

${ }^{14}$ In terms of rationality the players who have lost all their valuable belongings have nothing to lose. It would be the same to respawn. Therefore players tend to fight to the end or try to flee rather than surrender. Also bandits prefer killing their victims rather than robbing them, because it less risky and faster.

${ }^{15}$ Hobbes, Leviathan, XIII.8.

${ }^{16}$ Hobbes, Leviathan, XIII.9.

${ }^{17}$ Hobbes, Leviathan, XIII.13.

${ }^{18}$ Hobbes, Leviathan, XVII.15.

${ }^{19}$ Hobbes, Leviathan, XIII.

${ }^{20}$ In the early alpha stages of the mod players were generally more trusting towards each other, but as the kill-on-sight mentality became the norm the community grew more suspicious. There was evident clash between the conventions of cooperative- and player versus player (pvp) -oriented FPS traditions. Arma 2 was a cooperative military simulator, but DayZ replaced teamwork with pvp.

21 Todd K. Platts, 'Locating Zombies in the Sociology of Popular Culture,' Sociology Compass 7 (2013), 547.

${ }^{22}$ Katie Salen and Eric Zimmerman, Rules of Play: Game Design Fundamentals (Cambridge: The MIT Press, 2003), 37.

${ }^{23}$ Across the genres moral decisions have been implemented to enhance narratives. Most of the games employing morality as plot device are single-player games, in which the effects of the choices made are more controllable. Often these choices are not dynamic, but reduced to few predefined possibilities. They tend to be artificial with little impact on how the game actually continues, for instance scripted to cutscenes that limit player agency.

${ }^{24}$ Ian Bogost (2007) coined the term "procedural rhetoric" to describe rhetoric that is conveyed through running processes, rules and mechanics. 
Spec Ops: The Line (2012) has adopted similar approach in its treatment of potentially controversial theme.

\section{Bibliography}

Bishop, Kyle. 'Raising the Dead: Unearthing the Non-Literary Origins of Zombie Cinema.' Journal of Popular Film and Television 33.4 (2006): 196-205.

Bogost, Ian. Persuasive Games: The Expressive Power of Video Games. Cambridge: The MIT Press, 2007.

Frasca, Gonzalo. 'Ephemeral Games: Is It Barbaric to Design Videogames after Auschwitz.' Cybertext Yearbook 2000, edited by Markku Eskelinen and Raine Koskimaa, 172-181. Jyväskylä: University of Jyväskylä, 2001.

Hobbes, Thomas. Leviathan or the Matter, Forme \& Power of the Common-Wealth and Civill. 1651.

Lauro, Sarah Juliet and Karen Embry. 'A Zombie Manifesto: The Nonhuman Condition in the Era of Advanced Capitalism.' Boundaries 2 35.1 (2008): 85-108.

Carter, Marcus, Martin Gibbs and Greg Wadley, 'Death and Dying in DayZ.' Proceedings of the $9^{\text {th }}$ Australasian Conference on Interactive Entertainment: Matters of Life and Death, Article No. 22. 1-6. New York: ACM, 2013.

Platts, Todd K. 'Locating Zombies in the Sociology of Popular Culture.' Sociology Compass 7 (2013): 547-560.

Ruch, Adam. 'Stockholm Syndrome: How Six Men Kidnapped Me in DayZ.' Games On Net, 5 July 2012. Viewed 16 May 2014. http://games.on.net/2012/07/stockholm-syndrome-how-six-men-kidnapped-me-indayz/

Salen, Katie and Eric Zimmerman. Rules of Play: Game Design Fundamentals. Cambridge: The MIT Press. 2003.

Tero Pasanen is a Ph.D student at University of Jyväskylä (JyU), Finland. His forthcoming dissertation examines how game-related controversies and moral panics have shaped the societal status of digital games. 